\title{
Implantes em pacientes com osteonecrose dos maxilares associado ao uso de bifosfonatos. Relato de caso e revisão de literatura
}

Implants in patients with bisphosphonate-related osteonecrosis of the jaw. Clinical case and literature review Implantes en pacientes con osteonecrosis de los maxilares inducida por bifosfonatos. Reporte de caso y revisión de literatura

\author{
Juliana Zorzi COLÉTE \\ Henrique HADAD 1 \\ Gustavo Antônio Correa MOMESSO1 \\ Hélio dos SANTOS FILHO2 \\ Breno dos Reis FERNANDES 1 \\ Idelmo Rangel GARCIA JÚNIOR ${ }^{3}$
} 'Programa de Pós-Graduação em Odontologia, Faculdade de Odontologia de Araçatuba, UNESP - Univ. Estadual Paulista, 16015-050 Araçatuba-SP, Brasil
'Cirurgião-Dentista, especialista em Implantodontia, 16015-050 Araçatuba-SP, Brasil

${ }^{3}$ Departamento de Cirurgia e Clínica Integrada, Faculdade de Odontologia de Araçatuba, UNESP - Univ. Estadual Paulista, 16015-050 Araçatuba-SP, Brasil

\section{Resumo}

A osteoporose é responsável por causar condições devastadoras no tecido ósseo elevando o risco de fraturas, constituindo uma problemática importante de saúde pública. É comum o uso de medicamentos, como os bifosfonatos para o controle dessa patologia. Contudo, a associação do uso desse medicamento com a osteonecrose dos maxilares vem sendo amplamente discutido na literatura, assim como a dificuldade da reabilitação desses pacientes como implantes e do protocolo de tratamento dessa condição. Assim sendo, o objetivo do presente trabalho foi realizar uma revisão de literatura para discutir as principais falhas associadas a instalação de implantes em pacientes portadores de osteonecrose dos maxilares associada ao uso de bifosfonatos (OMAB), assim como as possibilidades de tratamento, e relatar um caso clínico. As informações obtidas na revisão nos permitiu concluir que o uso de bifosfonatos orais, como o alendronato, é capaz de levar ao desenvolvimento da $\mathrm{OMAB}$ sendo necessária bastante precaução na reabilitação oral com implantes dentários, tanto em pacientes que fazem uso, como pacientes que apresentam risco de futura utilização de bifosfonatos para o tratamento de desordens esqueléticas.

Descritores: Osteoporose; Alendronato; Osteonecrose; Implantes Dentários.

\section{Abstract}

Osteoporosis is responsible for the devastating disease in the bone tissue, increasing the risk of fractures, constituting an important public health problem. It is common to use medications, such as bisphosphonates to control this pathology. The dosage application with osteonecrosis of the jaws has been discussed in the literature, as well as a rehabilitation task such as the implant and the transport protocol of this condition. Thus, the present study was carried out in a literature review on the main problems related to bisphosphonate-related osteonecrosis of the jaw (BRONJ) as well as the possibilities of treatment, and to report a clinical case. The information can be reviewed together, so that the use of oral bisphosphonates, such as alendronate, will be able to lead to the development of bisphosphonate-related osteonecrosis of the jaw (BRONJ), the future use of bisphosphonates for the treatment of skeletal disorders.

Descriptors: Osteoporosis; Alendronate; Osteonecrosis; Dental Implants.

\section{Resumen}

La osteoporosis es responsable de causar condiciones devastadoras en el tejido óseo, elevando el riesgo de fracturas, constituyendo una problemática importante de salud pública. Es común el uso de medicamentos, como los bifosfonatos para el control de esa patología. Sin embargo, la asociación de los usos de este medicamento con la osteonecrosis de los maxilares viene siendo ampliamente discutida en la literatura, así como la dificultad de la rehabilitación dos pacientes como implantes y del protocolo de tramo de esa condición. Por lo tanto, el objetivo del presente trabajo fue realizar una revisión de literatura para discutir las principales fallas asociadas a la instalación de implantes en pacientes portadores de osteonecrosis maxilar inducida por bifosfonatos (ONMB), así como las posibilidades de tratamiento, e informar un caso clínico. La información obtenida en la revisión nos permitió concluir que el uso de bifosfonatos orales, como el alendronato, es capaz de llevar al desarrollo de la osteonecrosis maxilar inducida por bifosfonatos (ONMB), siendo necesario bastante precaución en la rehabilitación oral con implantes dentales, tanto en pacientes que hacen uso, como pacientes que presentan riesgo de la futura utilización de bifosfonatos para el tratamiento de desórdenes esqueléticos.

Descriptores: Osteoporosis; Alendronato; Osteonecrosis; Implantes Dentales.

\section{INTRODUÇÃO}

Cada vez mais vem sendo relatado na literatura casos de falhas na instalação de implantes dentários. Essas falhas possuem diversas etiologias e podem se estabelecer em diferentes momentos, sendo classificadas em recentes ou tardias ${ }^{1-3}$.

Os bifosfonatos (BF's) representam uma classe de medicamentos inibidores da atividade osteoclástica e são considerados primeira opção no tratamento de doenças que afetam o metabolismo ósseo, tais como, osteoporose, doença de Paget, além de tumores malignos, como mieloma múltiplo, hipercalcemia maligna e metástases ósseas ${ }^{4-6}$. O alendronato é o BF mais indicado para o tratamento de osteoporose pós menopáusica e aprovado pela food and drug administration (FDA) sendo indiscutível sua redução nas fraturas vertebrais e de ossos longos ${ }^{7,8}$.
Apesar dos benefícios dos BF's no tratamento de desordens esqueléticas, em 2003 foram descritos os primeiros casos de osteonecrose dos maxilares associado ao uso de bifosfonatos (OMAB) que se apresenta clinicamente como uma área de exposição óssea com presença de necrose e secreção purulenta que não sofre reparação com mais de 8 semanas em pacientes que fazem uso prévio ou atual de bifosfonatos sem história prévia de radioterapia na região de cabeça e pescoço ${ }^{9-11}$. A Associação Americana de Cirurgiões Orais e Maxilofaciais (AAOMS) descreveram que a osteonecrose dos maxilares pode ocorrer também através do uso de outros medicamentos antirreabsortivos que não os BF's, mudando a nomenclatura da doença para Osteonecrose dos maxilares associada ao uso de medicamentos $(\mathrm{OMAM})^{12}$. 
O aparecimento da OMAB está mais comumente associado a pacientes que fazem uso de ácido zoledrônico, administrado via intravenosa, para o tratamento de tumores e metástases ósseas ${ }^{13}$. No entanto pacientes que fazem uso de bifosfonatos orais, como o alendronato, amplamente prescrito para a prevenção e tratamento de osteoporose, também estão propensos para o desenvolvimento da doença, ainda que em menor proporção, recentes estudos indicam que seu aparecimento é maior do que se pensava anteriormente ${ }^{14}$. Apesar de o aparecimento espontâneo da OMAB ter sido relatado, a realização de procedimentos cirúrgicos na cavidade bucal, especialmente exodontias e instalação de implantes dentários, associados ao uso do medicamento, são fatores potenciais para que ocorra a condição ${ }^{15}$.

Dessa forma, o objetivo deste estudo foi realizar uma revisão de literatura sobre o uso de bifosfonatos e a ocorrência da osteonecrose dos maxilares e apresentar um caso clínico sobre esta condição, seu tratamento e a apresentar a nova reabilitação com implantes.

\section{REVISÃO DA LITERATURA}

\section{- Tratamento da osteoporose}

A osteoporose é responsável por causar condições devastadoras no tecido ósseo elevando o risco de fraturas, constituindo uma problemática importante de saúde pública. Mulheres e homens com mais de 50 anos representam, respectivamente, $50 \%$ e $20 \%$ da população caucasiana que irá apresentar alguma fratura devido à fragilidade óssea, ao longo da vida ${ }^{16}$. Na última década os bifosfonatos passaram a ser amplamente utilizados no tratamento da osteoporose e estudos clínicos evidenciaram que esses medicamentos são extremamente eficazes na redução de fraturas vertebrais (40-50\%) e não vertebrais (20-40\%), inclusive fraturas de costela ${ }^{17,18}$. Por este motivo, os bifosfonatos orais são os mais comumente prescritos em todo o mundo ${ }^{18}$.

\section{- Farmacologia dos bifosfonatos}

Os bifosfonatos (BF's) são análogos do pirofosfato inorgânico apresentando substituição de um átomo de carbono por um de oxigênio. É sabido, no entanto, que os pirofosfatos são reguladores naturais e fisiológicos do remodelamento ósseo, porém, quando ingeridos via oral sofrem degradação no trato gastrointestinal, tornando-se incapazes de inibir a calcificação óssea. Esses medicamentos detêm alta afinidade pelos tecidos mineralizados e atuam nos sítios em que ocorre o remodelamento ósseo ${ }^{19}$. Os BF's podem ser administrados via oral ou intravenosa, sendo $50 \%$ da droga absorvida pelo tecido ósseo e o restante excretado pelos rins ${ }^{20}$.

Observou-se, então, que essas drogas eram capazes de suprimir a reabsorção óssea sendo muito bem empregadas, em tratamentos de osteogênese imperfeita, osteoporose, displasia fibrosa, doença de
Paget, bem como em tumores metástases ósseas ${ }^{10,19}$. Além dessa característica, os BF's também proporcionavam redução da dor e aparecimento de lesões e fraturas ósseas, aumentando, significativamente, a qualidade de vida dos pacientes $^{21,22}$.

O mecanismo de ação dos BF's baseia-se na inibição da diferenciação celular das células precursoras dos osteoclastos e indução a apoptose. Outra característica de bastante importância dos BF's é sua capacidade antiangiogênica, reduzindo o número de vasos sanguíneos e a resposta endotelial aos hormônios angiogênicos ${ }^{10,23,24}$. Dentre os BF's mais utilizados, destaca-se o alendronato (10mg), administrado via oral e amplamente prescrito para o tratamento de osteoporose, eficaz no aumento da densidade óssea e diminuição de fraturas patológicas. Já entre os BF's administrados via intravenosa, destacam-se o pamidronato (90mg), de segunda geração, e o zoledronato $(4 \mathrm{mg})$ de terceira geração mais utilizado no tratamento de tumores e metástases ósseas ${ }^{25-27}$.

- Osteonecrose dos maxilares associada ao uso de bifosfonatos (OMAB)

Apesar de os BF's serem extremamente benéficos no tratamento das desordens esqueléticas, $\mathrm{o}$ uso a longo prazo deste medicamento pode levar ao desenvolvimento de um importante efeito colateral chamado osteonecrose dos maxilares associado ao uso de bifosfonatos (OMAB $)^{9,10,13}$. Diversos casos estão sendo descritos, desde 2003 associando o uso de bifosfonatos e o aparecimento de áreas de necrose óssea na região dos maxilares ${ }^{5,10,28-35}$.

- Fisiopatologia da OMAB

A patogênese da OMAB ainda é incerta, no entanto muitas propostas têm sido descritas. A maior parte dos autores acredita que se trata do desequilíbrio no remodelamento fisiológico dos ossos maxilares ou reparo alveolar. A supressão excessiva dos osteoclastos prejudica o turnover ósseo de modo que os microtraumas causados ao longo da vida, bem como injúrias no tecido ósseo decorrentes de exodontias ou instalação de implantes, impedem que haja reparação tecidual, resultando em necrose óssea ${ }^{13}$.

Alguns bifosfonatos, como o ácido zoledrônico, podem apresentar efeitos antiangiogênicos através da diminuição dos níveis do fator de crescimento endotelial na circulação ${ }^{36,37}$, levando à diminuição do suprimento sanguíneo para o tecido ósseo local, contribuindo para o aparecimento de áreas isquêmicas, observadas nos pacientes afetados. A combinação de todos esses fatores pode desencadear o aparecimento da OMAB. A predileção pelos ossos maxilares em relação a outras regiões do esqueleto humano pode ser devido ao fato de que os BF's são depositados em regiões de intenso remodelamento ósseo, como a região 
craniofacial. No entanto, sabe-se que é necessária a realização de mais estudos que validem todas as suposições que existem em torno da patogênese da $\mathrm{OMAB}^{13}$.

Diversos estudos clínicos retrospectivos evidenciaram que o desenvolvimento da OMAB pode estar ligado a fatores de risco, tais como, traumas dento-alveolares, duração do tratamento com BF's e o tipo de BF administrado ${ }^{5,38-40}$. De acordo com os relatos clínicos publicados até o momento, o trauma dento-alveolar recente parece ser o fator de risco mais prevalente para o surgimento da $\mathrm{OMAB}^{30,38}$. Os BF's intravenosos, como o Pamidronato e, especialmente, o ácido zoledrônico, apresentam maior incidência do quadro quando comparados aos BF's orais. No entanto, o número de casos de OMAB associado ao uso de BF's orais tem sido cada vez maior tornando-se também, motivo de preocupação $^{10,30,41}$.

A Associação Americana de Cirurgiões Orais e Maxilofaciais (AAOMS) salienta que a OMAB pode ocorrer em ambos os sexos, no entanto há predileção pelo gênero feminino, já que o uso de BF's é amplamente indicado no tratamento de doenças como câncer de mama e osteoporose que acometem predominantemente mulheres. $\mathrm{O}$ desenvolvimento de OMAB em pacientes que fazem uso de BF's orais para o tratamento de osteoporose ocorre depois de longa exposição do paciente ao medicamento (geralmente mais de 3 anos), ou na terapia concomitante com esteroides ${ }^{13}$.

Em 2005, a FDA posicionou-se frente ao crescimento no número de casos de OMAB através de uma nota, alertando para o risco no uso de todos os bifosfonatos, provocando mudanças na prática clínica de prescrição desta classe de medicamentos, já que as benesses da droga na prevenção de fraturas osteoporóticas eram cada vez mais observadas ${ }^{42}$.

\section{- Tratamento da OMAB}

Uma vez que a condição é instalada, torna-se um grande desafio estabelecer o protocolo de tratamento para sua erradicação. Segundo Ruggiero ${ }^{13}$ para pacientes que estão iniciando o tratamento com BF's não há nenhum procedimento específico, a não ser a orientação para manutenção da saúde bucal e acurado exame clínico no intuito de descobrir possíveis alterações que necessitem de tratamento cirúrgico. $\mathrm{O}$ autor acrescenta que em pacientes que fazem uso de $\mathrm{BF}$, mas que não apresentam OMAB, está contra indicada a realização de cirurgias bucais, além da necessidade de reembasamento de próteses mucossuportadas. Ruggiero ${ }^{13}$ aponta ainda que para pacientes que são portadores de OMAB, o plano de tratamento é mais complexo, uma vez que já desenvolveu a condição patológica. Assim, foi estabelecido um protocolo de tratamento para esses pacientes, baseado no estágio clínico da doença. O estágio 1, caracterizado pela presença de osso exposto/necrótico, sem sintomatologia de infecção, o tratamento é baseado na prescrição de bochechos antibacterianos e acompanhamento clínico a cada 3-4 meses, além de orientações ao paciente. No estágio 2, caracterizado pela presença de osso exposto/necrótico com presença de infecção, evidenciada por dor e eritema na região, o tratamento envolve a prescrição de bochechos antibacterianos e antimicrobianos, como clindamicina, penicilina, fluorquinolonas, metronidazol e controle da dor. Para o estágio 3, caracterizado por apresentar maior agressividade pois além de agrupar todas as características dos estágios anteriores, pode acompanhar fraturas patológicas, fístulas extraorais e osteólise estendendo para a base mandibular, o tratamento consiste em realizar os protocolos anteriores com o aditivo de que neste estágio é indicado realizar o debridamento e ressecção cirúrgica quando necessário, para controle da infecção e dor ${ }^{13}$.

\section{CASO CLÍNICO}

Paciente do gênero feminino, caucasiana, 58 anos, em 2005 foi submetida à instalação de implantes osseointegráveis, sendo dois em maxila e cinco em mandibula para suportar uma prótese protocolo de Branemark. Após o período de osseointegração foi realizada a instalação da prótese protocolo inferior e a prótese overdenture superior, finalizando a reabilitação da paciente (Figura1).

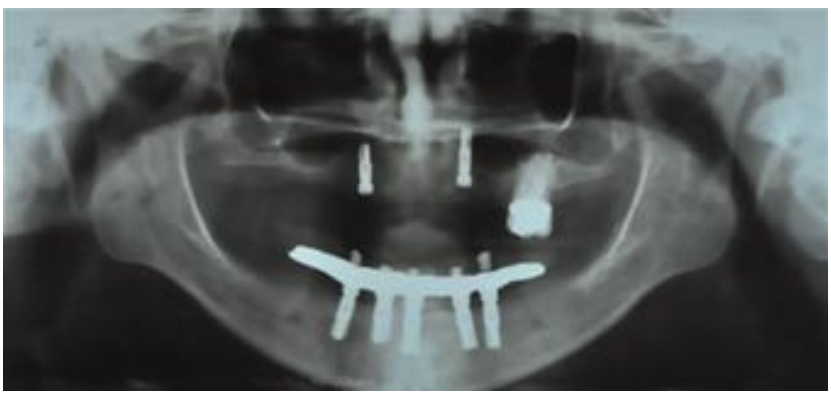

Figura 1: Radiografia panorâmica da primeira reabilitação da paciente com implantes osseointegráveis em 2005.

Após cinco anos da instalação dos implantes a paciente passou por exames periódicos sobre sua condição óssea, com diagnóstico de osteopenia. A paciente foi submetida à terapia com alendronato de sódio $70 \mathrm{mg}$, reposição hormonal com Totelli e reposição com cálcio de ostra $500 \mathrm{mg}$ para prevenção de osteoporose. Em 2012, sete anos após a instalação dos implantes, ao se alimentar, a paciente notou um barulho estranho, sensação dolorosa e fratura da prótese dentária. Foi encaminhada à equipe e, ao exame clínico observou-se fratura da barra metálica inferior sendo ela removida imediatamente. Além disso, constatou-se a presença de halitose provindo da região dos implantes e discreta exposição óssea mandibular (Figura 2).

Foi solicitada nova radiografia panorâmica, evidenciando a presença de perda óssea severa na região peri-implantar, no entanto sem presença de 
sequestro ósseo (Figura 3A e 3B). A partir dessas características clínicas, foi questionado à paciente se ela estava fazendo uso de algum medicamento novo, não relatado previamente. A paciente informou que há dois anos foi diagnosticada com osteopenia e iniciou terapia medicamentosa com reposição de cálcio e alendronato de sódio. Diante desta informação e somado as características clínicas, fechou-se o diagnóstico de OMAB.

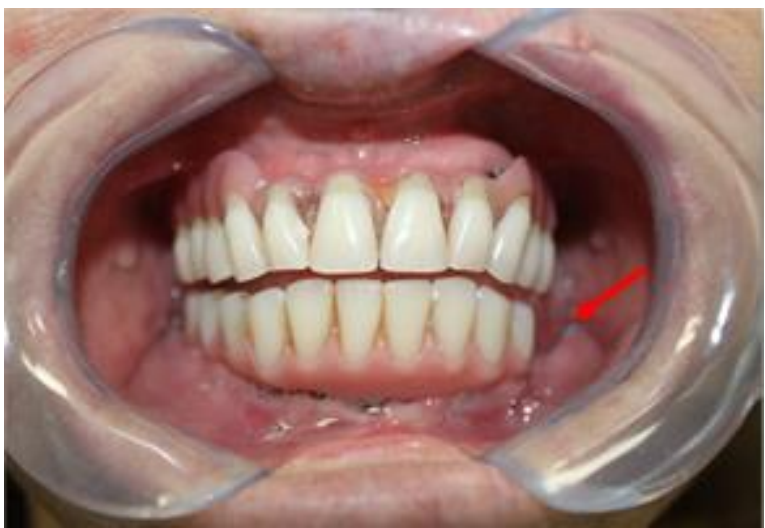

Figura 2: Região fraturada da barra metálica (seta) e prótese sobre implante no qual ocorreu exposição de tecido ósseo necrótico
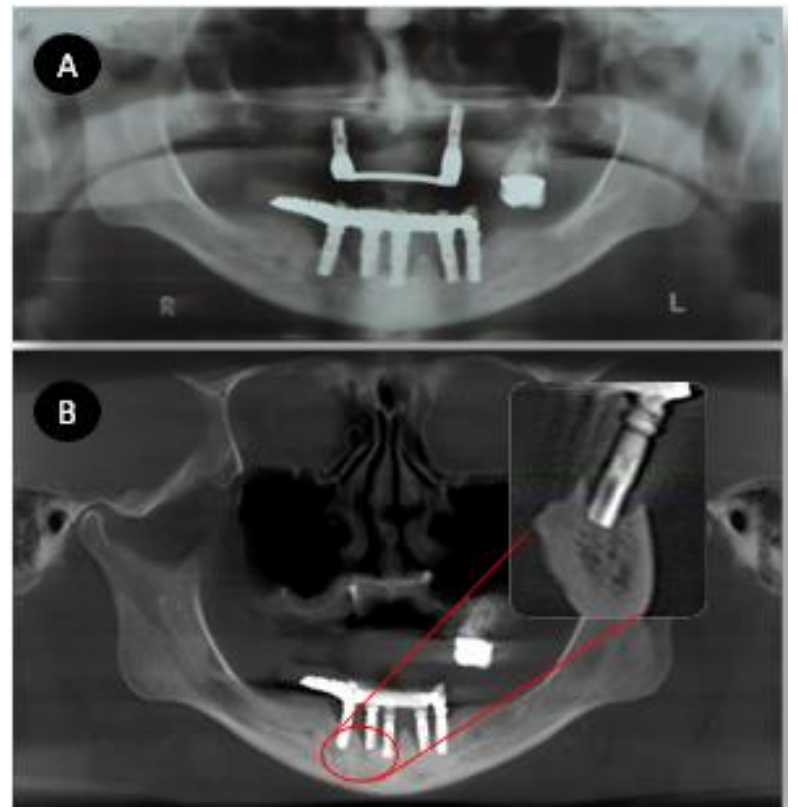

Figura 3: A.Radiografia panorâmica evidenciando osteólise periimplantar e perda dos implantes. B.Tomografia computadorizada de mandíbula com aproximação comprovando a osteólise periimplantar devido à presença de osteonecrose.

$\mathrm{O}$ tratamento consistiu em fazer, primeiramente, a remoção dos implantes. No momento da remoção foi possível observar que a prótese cimentada e os implantes estavam unidos como um bloco, apresentando bridas de osso necrótico em torno do implante, devido à extensa perda óssea peri-implantar (Figura 4A e B).

Inicialmente optou-se por um tratamento mais conservador e estipulou-se, como plano de tratamento para a resolução da osteonecrose, o protocolo para o estágio 1 de Ruggiero ${ }^{13}$, já que a paciente não apresentava sinais clínicos de infecção ou osteólise excessiva. Foi estabelecido, então, deixar o sítio da lesão aberto e aplicar PVPI tópico a 10\% na região por meio de gaze estéril. Associado a isso, foram prescritos bochechos com clorexidina a $0,12 \%$ três vezes ao dia e Clindamicina $300 \mathrm{mg}$, via oral, de 8/8 horas, durante 10 dias. Após sete dias de tratamento observou-se melhora no aspecto cicatricial e como conduta realizou-se a sutura do sítio previamente aberto mantendo o antibiótico e bochechos até o fim da terapia. Após 14 dias notouse bom aspecto cicatricial da sutura, sem presença de deiscência ou exposição óssea.
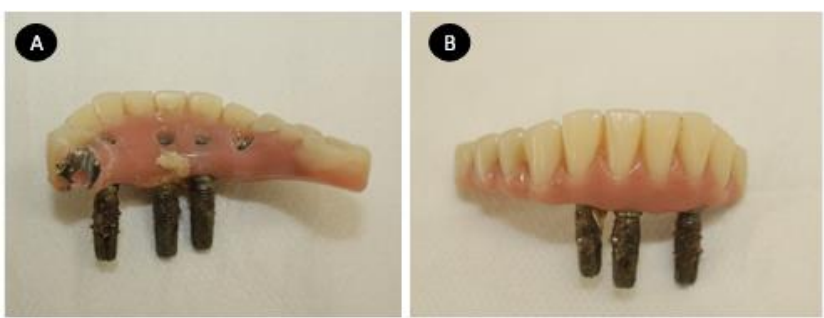

Figura 4: A.Prótese sobre implante retirada da paciente em corpo único, em razão da grande perda óssea peri-implantar. B.Presença de tecido ósseo necrótico na superfície dos implantes.

Foi realizado acompanhamento periódico com a paciente para observar possíveis recidivas da lesão e realizou-se a reabilitação com prótese mucossuportada, superior e inferior adaptada de modo que não causasse traumas na região, os quais poderiam levar à nova exposição óssea e infecção da região. Após o desenvolvimento da OMAB, entramos em contato com o médico responsável pelo tratamento para discutir sobre a necessidade de continuar a terapia com o alendronato. Por ter sido prescrita apenas para prevenção, foi interrompido o uso da droga pelo médico. Dois anos após a resolução da OMAB optou-se por reabilitar a paciente com 2 implantes maxilares e 2 mandibulares que conseguimos manter, suportados por próteses overdenture, devido a queixas sobre o uso de próteses mucossuportadas (Figura 5). Obteve-se assim não só êxito no tratamento da OMAB, como também satisfação da paciente com as novas próteses instaladas.

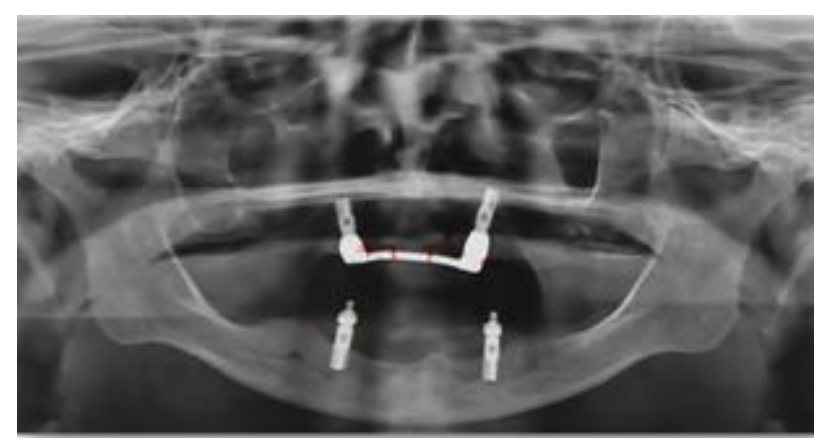

Figura 5: Radiografia panorâmica dois anos após suspensão da terapia com alendronato e resolução da OMAB, mostrando reabilitação final da paciente com prótese dentária suportada por implantes dentários. 


\section{DISCUSSÃO}

Os fatores de risco mais relevantes para o desenvolvimento de OMAB são, sabidamente, a realização de procedimentos cirúrgicos na cavidade bucal. Além disso, a maioria dos casos de OMAB associados à instalação de implantes dentários envolvem pacientes que são submetidos à terapia com o bifosfonato nitrogenado ácido zoledrônico, para o tratamento e metástases ósseas e mieloma múltiplo ${ }^{9,10,43}$. Dessa forma, este tipo de cirurgia está absolutamente contraindicada em pacientes que fazem uso de ácido zolerdrônico ${ }^{11,44}$.

A correlação da OMAB em pacientes que fazem uso de bifosfonatos orais e submetidos à instalação de implantes dentários tem sido pouco descrita e demonstra ser bem inferior à quantidade de casos relacionados à terapia com ácido zoledrônico $(0.01 \%-0.04 \% \text { versus } 0.7 \%-12 \%)^{41,45}$.

A diferença na incidência da OMAB entre os diferentes medicamentos utilizados pode ser explicada por alguns fatores como o tipo de bifosfonato, via de administração (oral ou intravenosa), duração do tratamento e dose acumulativa $^{46}$. Além disso, a potência dos BF's orais é bastante inferior aos intravenosos, sendo um fator indicativo da menor incidência de $O M A B$ nesse primeiro grupo.

No entanto, apesar dos dados indicando a menor incidência de OMAB em pacientes submetidos à terapia com alendronato, não é correto afirmar que essa condição patológica não ocorre nesse grupo de pacientes, uma vez que diversos estudos têm demonstrado perdas e falhas na instalação de implantes dentários associado ao uso de alendronato $^{43,47-49}$. Em uma série de casos, LopezCedrun et al. ${ }^{49}$ avaliaram 9 pacientes com OMAB associado à instalação de implantes dentários e observaram que o alendronato foi o medicamento mais frequente. Esse dado pode ser confirmado, também, por outros estudos clínicos ${ }^{49,50}$. Os mesmos autores evidenciaram, ainda, que, de 101 pacientes que apresentaram OMAB, 59\% fazia uso de BF intravenoso, no entanto, $41 \%$ eram submetidos à terapia com BF oral, não denotando diferença significativa. Portanto, é possível observar que apesar da menor potência, o alendronato apresenta ser importante fator de risco para o desenvolvimento de $\mathrm{OMAB}^{47}$.

Além disso, em estudo experimental desenvolvido por Ramalho-Ferreira et al. $^{51}$ é comprovado através da análise por microscopia confocal de dinâmica óssea (fluorocromos) que, apesar do efeito antirreabsortivo do alendronato, o medicamento suprime o turnover ósseo de forma tão intensa que, à longo prazo, não há mais presença de renovação óssea restando apenas osso velho na região periimplantar ${ }^{51}$.

Nosso estudo relata um caso incomum de
OMAB na qual a paciente procurou a reabilitação com implantes dentários sem apresentar qualquer contraindicação para tal. No entanto, 5 anos após o procedimento cirúrgico foi diagnosticada com osteopenia sendo submetida à terapia com alendronato de sódio como prevenção para osteoporose. Dois anos após o início da terapia medicamentosa, a paciente apresentou falha na osseointegração e perda dos implantes dentários mandibulares, além de perda óssea considerável na região periimplantar. É sabido que o desenvolvimento de OMAB é mais comum em casos em que a reabilitação com implantes dentários é realizada após um tempo do início da terapia com o bifosfonato, devido à impossibilidade do sucesso na osseointegração, uma vez que o remodelamento e o turnover ósseo estão suprimidos. Segundo os guidelines da AAOMS, em pacientes que fazem uso de BF oral por mais de três anos, quando suspendido o uso do medicamento três meses antes e após a realização de procedimento cirúrgico oral, há redução do risco de desenvolvimento de $\mathrm{OMAB}^{11}$. A suspensão do $\mathrm{BF}$ oral tende a normalizar a função osteoclástica e o turnover ósseo em 3 meses, elevando os marcadores para o remodelamento ósseo total $^{52}$. No entanto essa premissa não é totalmente verdadeira para os ossos maxilares, sendo bastante questionável a liberação para a cirurgia bucal, uma vez que mesmo que os implantes já estejam osseointegrados, o tecido ósseo está em constante remodelação e é submetido à microtraumas constantemente, principalmente em se tratando dos ossos gnáticos, que suportam a carga mastigatória ${ }^{53}$. Portanto, há muitas controvérsias em relação à aplicação do "drug holiday" para pacientes candidatos à reabilitação com implantes dentários que fazem uso de BF's orais. Sendo assim, é possível que a OMAB tenha se desenvolvido em nossa paciente de acordo com esse princípio, mesmo após cinco anos da reabilitação maxilofacial.

Outro ponto importante a ser abordado é que os pacientes que desenvolvem OMAB decorrente do tratamento com BF's orais respondem melhor aos tratamentos propostos. $\mathrm{O}$ tratamento de pacientes portadores de OMAB variam de acordo com a evolução do estágio clínico do paciente ${ }^{48}$. Ruggiero $^{11,13}$ propôs 3 estágios de evolução para a OMAB, bem como os tratamentos de acordo com cada fase.

A paciente referente ao nosso estudo apresentou falha na osseointegração devido à osteólise na região peri-implantar, exposição de osso necrótico, no entanto sem presença de infecção. As características clínicas observadas são compatíveis com o estágio 1 de Ruggiero ${ }^{11,13}$. O tratamento proposto para esta condição clínica inclui a prescrição de bochechos antimicrobianos, antibiótico e orientações ao paciente. Baseando-se nesse 
protocolo clínico, foi prescrito bochecho com clorexidina $0,12 \%$ três vezes ao dia, clindamicina $300 \mathrm{mg}$ três vezes ao dia, por sete dias. Adicionalmente a este protocolo, optamos por orientar a paciente a realizar a limpeza do sítio afetado através da fricção de uma gaze embebida em PVPI $10 \%$ associado ao bochecho, durante sete dias. $\mathrm{O}$ tratamento proposto apresentou resultados favoráveis com reparação total lesão.

Além do correto tratamento da OMAB, a dúvida que permanece entre os cirurgiões bucomaxilofaciais é como reabilitar o paciente após o tratamento da osteonecrose mandibular. É sabido que a meia vida plasmática dos $\mathrm{BF}$ 's é longa permanecendo no tecido ósseo por volta de 10 anos ${ }^{11}$. Por este motivo há muita discussão em torno da suspensão do medicamento (drug holiday) para a reabilitação com implantes dentários retomando a terapia posteriormente ou quanto tempo de espera seria necessário para reabilitar o paciente após a suspensão completa do medicamento. Os dados ainda são bastante controversos, porém, maior parte dos autores tendem a pensar que o drug holiday é uma alternativa viável para pacientes que fazem uso de BF's orais ${ }^{11,52}$, no entanto a FDA publicou nota alertando para o fato de não haver nenhuma evidência sobre quando iniciar ou quanto tempo deve durar o "drug holiday". Nosso estudo comprova que os BF's podem ser imprevisíveis neste ponto. Ainda que os exames do paciente deem sinais de que há melhora nos marcadores de remodelação óssea, os BF's são medicamentos que causam efeitos devastadores no remodelamento ósseo, podendo levar a perda de implantes devidamente osseointegrados, ainda que seu uso seja iniciado cinco anos após o procedimento cirúrgico.

Apesar do desenvolvimento atípico de OMAB em nosso caso, dois anos após o tratamento condição e a suspensão da droga, a paciente foi submetida à reabilitação com implantes dentários suportados por uma prótese overdenture, obtendo sucesso na osseointegração e função dos mesmos, denotando que a suspensão da droga foi fator decisivo no sucesso da reabilitação oral. Este sucesso foi obtido, muito possivelmente, pelo fato de que a paciente fez uso de um BF de menor potência (alendronato) por pouco tempo (2 anos) e foi tratada no estágio inicial da doença.

Muitos estudos ainda devem ser realizados em torno da problemática da OMAB para que se chegue a um consenso sobre sua correta patogênese, qual o tratamento mais adequado e, principalmente, em busca de estratégias preventivas, como a parceria entre médicos e cirurgiões bucomaxilofaciais no plano de tratamento dos pacientes, bem como, na possível utilização de novos medicamentos para o tratamento e prevenção da osteoporose, como o raloxifeno e o ranelato de estrôncio, tão eficientes quanto o alendronato e não relacionados ao desenvolvimento de $\mathrm{OMAB}^{54,55}$.

\section{CONCLUSÃO}

Dessa forma, podemos concluir que os bifosfonatos orais, como o alendronato são capazes de levar ao desenvolvimento da OMAB sendo necessária bastante precaução na reabilitação oral com implantes dentários, tanto em pacientes que fazem uso, como em pacientes que apresentam risco de futura utilização da droga para o tratamento de desordens esqueléticas.

\section{REFERÊNCIAS}

1. Esposito M, Hirsch JM, Lekholm U, Thomsen P. Biological factors contributing to failures of osseointegrated oral implants. (I). Success criteria and epidemiology. Eur J Oral Sci. 1998;106(1):527-51.

2. Quirynen M, De Soete M, Van Steenberghe D. Infectious risks for oral implants: a review of the literature. Clin Oral Implants Res. 2002;13(1):1-19.

3. Van Steenberghe D, Jacobs R, Desnyder M, Maffei G, Quirynen M. The relative impact of local and endogenous patient-related factors on implant failure up to the abutment stage. Clin Oral Implants Res. 2002;13(6):617-22.

4. Migliorati CA. Bisphosphanates and oral cavity avascular bone necrosis. J Clin Oncol. 2003;21(22)4253-54.

5. Migliorati CA, Casiglia J, Epstein J, Jacobsen PL, Siegel MA, Woo SB. Managing the care of patients with bisphosphonate-associated osteonecrosis: an American Academy of Oral Medicine position paper. J Am Dent Assoc. 2005;136(12):1658-68.

6. Madrid C, Sanz M. What impact do systemically administrated bisphosphonates have on oral implant therapy? A systematic review. Clin Oral Implants Res. 2009;20(Suppl 4):87-95.

7. Black DM, Schwartz AV, Ensrud KE, Cauley JA, Levis S, Quandt SA et al. Effects of continuing or stopping alendronate after 5 years of treatment: the Fracture Intervention Trial Long-term Extension (FLEX): a randomized trial. JAMA. 2006;296(24):2927-38.

8. Schwartz AV, Bauer DC, Cummings SR, Cauley JA, Ensrud KE, Palermo L et al. Efficacy of continued alendronate for fractures in women with and without prevalent vertebral fracture: the FLEX trial. J Bone Miner Res. 2010; 25(5):976-82.

9. Marx RE. Pamidronate (Aredia) and zoledronate (Zometa) induced avascular necrosis of the jaws: a growing epidemic. J Oral Maxillofac Surg. 2003;61(9):1115-17.

10.Ruggiero SL, Mehrotra B, Rosenberg TJ, Engroff SL. Osteonecrosis of the jaws associated with the use of bisphosphonates: a review of 63 
cases. Journal of oral and maxillofacial surgery, 2004;62(5):527-34.

11.Ruggiero SL. Guidelines for the diagnosis of bisphosphonate-related osteonecrosis of the jaw (BRONJ). Clin Cases Miner Bone Metab.2007;4(1):37-42.

12.Ruggiero SL, Dodson TB, Assael LA, Landesberg R, Marx RE, Mehrotra B et al. American Association of Oral and Maxillofacial Surgeons position paper on bisphosphonate-related osteonecrosis of the jaws-2009 update. J Oral Maxillofac Surg. 2009;67(5 Suppl):2-12.

13.Ruggiero SL, Drew SJ. Osteonecrosis of the jaws and bisphosphonate therapy. J Dent Res. 2007;86(11):1013-21.

14. Otto S, Abu-Id MH, Fedele S, Warnke PH, Becker ST, Kolk A et al. Osteoporosis and bisphosphonates-related osteonecrosis of the jaw: not just a sporadic coincidence-a multi-centre study. J Craniomaxillofac Surg. 2011; 39(4):272-77.

15. Yarom N, Yahalom R, Shoshani Y, Hamed W, Regev E, Elad S. Osteonecrosis of the jaw induced by orally administered bisphosphonates: incidence, clinical features, predisposing factors and treatment outcome. Osteoporos Int. 2007; 18(10):1363-70.

16.Sambrook P, Cooper C. Osteoporosis. Lancet. 2006;367(9527):2010-18.

17. Cranney A, Guyatt G, Griffith L, Wells G, Tugwell P, Rosen C. Meta-analyses of therapies for postmenopausal osteoporosis. Endocr Rev. 2002;23(4):570-78.

18. Close P, Neuprez A, Reginster JY. Developments in the pharmacotherapeutic management of osteoporosis. Expert Opin Pharmacother. 2006;7(12):1603-15.

19.Rodan GA, Fleisch HA. Bisphosphonates: mechanisms of action. J Clin Invest. 1996; 97(12): 2692-96.

20.Dannemann C, Grätz KW, Riener MO, Zwahlen RA. Jaw osteonecrosis related to bisphosphonate therapy: a severe secondary disorder. Bone. 2007;40(4):828-34.

21.Kos M, Luczak K, Godzinski J, Klempous J. Treatment of monostotic fibrous dysplasia with pamidronate. J Craniomaxillofac Surg. 2004; 32(1):10-15.

22.Pastor-Zuazaga D, Garatea-Crelgo J, MartinoGorbea R, Etayo-Pérez A, Sebastián-Lopez C. Osteonecrosis of the jaws and bisphosphonates. Report of three cases. Med Oral Patol Oral Cir Bucal. 2006;11(1):E76-9.

23. Sato M, Grasser W, Endo N, Akins R, Simmons $\mathrm{H}$, Thompson DD et al. Bisphosphonate action. Alendronate localization in rat bone and effects on osteoclast ultrastructure. J Clin Invest. 1991; 88(6):2095-2105.
24.Sahni M, Guenther HL, Fleisch H, Collin P, Martin TJ. Bisphosphonates act on rat bone resorption through the mediation of osteoblasts. J Clin Invest.1993;91(5):2004-11.

25.Merigo E, Manfredi M, Meleti M, Corradi D, Vescovi P. Jaw bone necrosis without previous dental extractions associated with the use of bisphosphonates (pamidronate and zoledronate): a four-case report. J Oral Pathol Med. 2005; 34(10):613-17.

26. Olson KB, Hellie CM, Pienta KJ. Osteonecrosis of jaw in patient with hormonerefractory prostate cancer treated with zoledronic acid. Urology. 2005;66(3):658.

27.Graziani F, Cei S, La Ferla F, Cerri E, Itro A, Gabriele M. Association between osteonecrosis of the jaws and chronic high-dosage intravenous bisphosphonates therapy. J Craniofac Surg. 2006;17(5):876-79.

28.Greenberg MS. Comment in. Intravenous bisphosphonates and osteonecrosis. Oral Surg Oral Med Oral Pathol Oral Radiol Endod. 2004;98(3):259-60.

29. Gibbs SD, O'Grady J, Seymour JF, Prince HM. Bisphosphonate-induced osteonecrosis of the jaw requires early detection and intervention. Med $\mathbf{J}$ Aust. 2005;183(10):549-50.

30.Marx RE, Sawatari Y, Fortin M, Broumand V. Bisphosphonate-induced exposed bone (osteonecrosis/osteopetrosis) of the jaws: risk factors, recognition, prevention, and treatment. J Oral Maxillofac Surg. 2005;63(11)1567-75.

31. Melo MD, Obeid G. Osteonecrosis of the jaws in patients with a history of receiving bisphosphonate therapy: strategies for prevention and early recognition. J Am Dent Assoc. 2005;136(12):1675-81.

32.Purcell PM, Boyd IW. Bisphosphonates and osteonecrosis of the jaw. Med J Aust. 2005; 182(8)417-18.

33. Vannucchi AM, Ficarra G, Antonioli E, Bosi A. Osteonecrosis of the jaw associated with zoledronate therapy in a patient with multiple myeloma. Br J Haematol. 2005;128(6):738.

34.Bilezikian JP. Osteonecrosis of the jaw - do bisphosphonates pose a risk? N Engl J Med. 2006;355(22):2278-81.

35. Van Poznak C, Estilo C. Osteonecrosis of the jaw. J Oncol Pract. 2006; 2(1):3-4.

36. Santini D, Vincenzi B, Avvisati G, Dicuonzo G, Battistoni F, Gavasci $M$ et al. Pamidronate induces modifications of circulating angiogenetic factors in cancer patients. Clin Cancer Res. 2002; 8(5):1080-84.

37. Wood J, Bonjean K, Ruetz S, Bellahcène A, Devy L, Foidart JM et al. Novel antiangiogenic effects of the bisphosphonate compound zoledronic acid. J Pharmacol Exp Ther. 2002;302(3):1055-61. 
38.Badros A, Weikel D, Salama A, Goloubeva O, Schneider A, Rapoport A et al. Osteonecrosis of the jaw in multiple myeloma patients: clinical features and risk factors. J Clin Oncol. 2006;24(6):945-52.

39. Woo SB, Hellstein JW, Kalmar JR. Narrative [corrected] review: bisphosphonates and osteonecrosis of the jaws. Ann Intern Med.2006;144(10):753-61.

40.Cafro AM, Barbarano L, Nosari AM, D'avanzo G, Nichelatti M, Bibas M et al. Osteonecrosis of the jaw in patients with multiple myeloma treated with bisphosphonates: definition and management of the risk related to zoledronic acid. Clin Lymphoma Myeloma. 2008;8(2):111-16.

41. Mavrokokki T, Cheng A, Stein B, Goss A. Nature and frequency of bisphosphonateassociated osteonecrosis of the jaws in Australia. $\mathbf{J}$ Oral Maxillofac Surg. 2007;65(3):415-23.

42. Odvina CV, Zerwekh JE, Rao DS, Maalouf N, Gottschalk FA, Pak CY. Severely suppressed bone turnover: a potential complication of alendronate therapy. J Clin Endocrinol Metab. 2005;90(3):1294-301.

43.Bedogni A, Blandamura S, Lokmic Z, Palumbo C, Ragazzo M, Ferrari $\mathrm{F}$ et al. Bisphosphonateassociated jawbone osteonecrosis: a correlation between imaging techniques and histopathology. Oral Surg Oral Med Oral Pathol Oral Radiol Endod. 2008;105(3):358-64.

44. Khan AA, Sándor GK, Dore E, Morrison AD, Alsahli M, Amin F et al. Canadian consensus practice guidelines for bisphosphonate associated osteonecrosis of the jaw. J Rheumatol. 2008; 35(7):1391-97.

45. Hoff AO, Toth BB, Altundag K, Johnson MM, Warneke $\mathrm{CL}, \mathrm{Hu} \mathrm{M}$ et al.Frequency and risk factors associated with osteonecrosis of the jaw in cancer patients treated with intravenous bisphosphonates. J Bone Miner Res. 2008; 23(6):826-36.

46. Assael LA. Oral bisphosphonates as a cause of bisphosphonate-related osteonecrosis of the jaws: clinical findings, assessment of risks, and preventive strategies. J Oral Maxillofac Surg. 2009;67(5 Suppl):35-43.

47.Lazarovici TS, Yahalom R, Taicher S, SchwartzArad D, Peleg O, Yarom N. Bisphosphonaterelated osteonecrosis of the jaw associated with dental implants. J Oral Maxillofac Surg. 2010;68(4):790-96.

48.Jacobsen C, Metzler P, Rössle M, Obwegeser J, Zemann W, Grätz KW. Osteopathology induced by bisphosphonates and dental implants: clinical observations. Clin Oral Investig. 2013;17(1):167-75.

49.López-Cedrún JL, Sanromán JF, García A, Peñarrocha M, Feijoo JF, Limeres J, Diz P. Oral bisphosphonate-related osteonecrosis of the jaws in dental implant patients: a case series. Br J Oral Maxillofac Surg. 2013;51(8):874-79.

50.Kwon TG, Lee CO, Park JW, Choi SY, Rijal G, Shin HI. Osteonecrosis associated with dental implants in patients undergoing bisphosphonate treatment. Clin Oral Implants Res. 2014; 25(5):632-40.

51.Ramalho-Ferreira G, Faverani LP, Prado FB, Garcia IR, Okamoto R. Raloxifene enhances periimplant bone healing in osteoporotic rats. Int $\mathbf{J}$ Oral Maxillofac Surg. 2015;44(6):798-805.

52.Bone HG, Hosking D, Devogelaer JP, Tucci JR, Emkey RD, Tonino RP. Ten years' experience with alendronate for osteoporosis in postmenopausal women. N Engl J Med. 2004; 350(12):1189-99.

53.Lerner UH. Bone remodeling in post-menopausal osteoporosis. J Dent Res. 2006;85(7):584-95.

54.Ettinger B, Black DM, Mitlak BH, Knickerbocker RK, Nickelsen T, Genant HK et al. Reduction of vertebral fracture risk in postmenopausal women with osteoporosis treated with raloxifene: results from a 3-year randomized clinical trial. JAMA. 1999;282(7):637-45.

55.Gallacher SJ, Dixon T. Impact of treatments for postmenopausal osteoporosis (bisphosphonates, parathyroid hormone, strontium ranelate, and denosumab) on bone quality: a systematic review. Calcif Tissue Int. 2010;87(6)469-84.

\section{CONFLITO DE INTERESSES}

Os autores declaram não haver conflitos de interesse.

\section{AUTOR PARA CORRESPONDENCIA}

\section{Henrique Hadad}

henriquehadad@gmail.com

Submetido em 09/06/2018 Aceito em 04/10/2018 